\title{
Finite edge-transitive oriented graphs of valency four: a global approach
}

\author{
Jehan A. Al-bar* Ahmad N. Al-kenani* Najat M. Muthana* \\ Cheryl E. Praeger*† Pablo Spiga ${ }^{\ddagger}$
}

Submitted: Oct 23, 2014; Accepted: Jan 4, 2016; Published: Jan 22, 2016

Mathematics Subject Classifications: 05C25;20B25

\begin{abstract}
We develop a new framework for analysing finite connected, oriented graphs of valency four, which admit a vertex-transitive and edge-transitive group of automorphisms preserving the edge orientation. We identify a sub-family of 'basic' graphs such that each graph of this type is a normal cover of at least one basic graph. The basic graphs either admit an edge-transitive group of automorphisms that is quasiprimitive or biquasiprimitive on vertices, or admit an (oriented or unoriented) cycle as a normal quotient. We anticipate that each of these additional properties will facilitate effective further analysis, and we demonstrate that this is so for the quasiprimitive basic graphs. Here we obtain strong restrictions on the group involved, and construct several infinite families of such graphs which, to our knowledge, are different from any recorded in the literature so far. Several open problems are posed in the paper.
\end{abstract}

Keywords: transitive group, orbital graphs and digraphs, oriented graph, quasiprimitive permutation group

\section{Introduction}

We initiate a new approach to studying finite connected oriented graphs of valency four, which admit a vertex-transitive and edge-transitive group of automorphisms preserving an edge orientation. We make a normal quotient reduction leading to what we call basic

\footnotetext{
*King Abdulaziz University, Jeddah, Saudi Arabia (jalbar@kau.edu.sa, jaal_bar@hotmail.com, analkenani@kau.edu.sa, aalkenani10@hotmail.com, nmuthana@kau.edu.sa, najat muthana@hotmail.com).

${ }^{\dagger}$ Centre for the Mathematics of Symmetry and Computation, School of Mathematics and Statistics, The University of Western Australia, 35 Stirling Highway, Crawley, WA 6009, Australia (cheryl.praeger@uwa.edu.au).

${ }_{\ddagger}^{\ddagger}$ University of Milano-Bicocca, 20125 Via Cozzi 55, Milano, Italy (pablo.spiga@unimib.it).
} 


\begin{tabular}{lll}
\hline Basic Type & Possible $\Gamma_{N}$ for $1 \neq N \triangleleft G$ & $\begin{array}{l}\text { Conditions on } G \text {-action } \\
\text { on vertices }\end{array}$ \\
\hline Quasiprimitive & $K_{1}$ only & quasiprimitive \\
Biquasiprimitive & $K_{1}$ and $K_{2}$ only $(\Gamma$ bipartite $)$ & biquasiprimitive \\
Cycle & at least one $C_{r}(r \geqslant 3)$ & at least one quotient action \\
& & $D_{2 r}$ or $Z_{r}$ \\
\hline
\end{tabular}

Table 1: Types of Basic pairs $(\Gamma, G)$ in $\mathcal{O G}(4)$

graphs which either admit quasiprimitive or biquasiprimitive actions on vertices, or are degenerate cycles (see Table 1 and the Framework discussion below). This new approach has been used before in other problems dealing with symmetries of graphs, and we believe that it will also bear fruit when studying oriented graphs, and in particular, half-arctransitive graphs of valency four.

\section{Oriented graphs.}

For a transitive permutation group $G$ on a set $X, \mathrm{D}$. G. Higman [17] realised the importance of the $G$-action induced on ordered point-pairs, namely, for $g \in G$ and $x, y \in X$, $g:(x, y) \mapsto\left(x^{g}, y^{g}\right)$ where $x^{g}$ denotes the image of $x \in X$ under the action of $g$. Apart from the diagonal $\{(x, x) \mid x \in X\}$, each $G$-orbit $\Delta$ in this induced action corresponds a graph $\mathcal{G}(\Delta)$ with vertex set $X$ admitting $G$ as a vertex-transitive and edge-transitive group of automorphisms. The edges of $\mathcal{G}(\Delta)$ are the unordered pairs $\{x, y\}$ for which at least one of $(x, y)$ and $(y, x)$ lies in $\Delta$; such ordered pairs are called the $\operatorname{arcs}$ of $\mathcal{G}(\Delta)$. If both $\operatorname{arcs}(x, y)$ and $(y, x)$ lie in $\Delta$ then $\Delta$ consists of all the arcs of $\mathcal{G}(\Delta)$ and $G$ acts arctransitively. All arc-transitive graphs arise in this way, and many classes of arc-transitive graphs have been studied intensively, such as distance transitive graphs $[4,42], s$-arctransitive graphs $[39,44]$, and locally primitive and locally quasiprimitive graphs $[21,41]$. If $G$ is not arc-transitive then $\Delta$ consists of exactly one of $(x, y)$ and $(y, x)$ for each edge $\{x, y\}$ of $\mathcal{G}(\Delta)$. Thus, by directing each edge $\{x, y\}$ from $x$ to $y$ if and only if $(x, y) \in \Delta$, the orbital graph $\mathcal{G}(\Delta)$ admits a $G$-invariant orientation of its edges and we say that the graph $\mathcal{G}(\Delta)$ is $G$-oriented. Notice that whenever $\mathcal{G}(\Delta)$ is $G$-oriented, the graph $\mathcal{G}(\Delta)$ has even valency, say $m$, that is, each vertex $x$ lies in exactly $m$ edges, with $m / 2$ of these edges $\{x, y\}$ directed from $x$ to $y$ and $m / 2$ with the opposite orientation. All graphs that admit a $G$-invariant orientation, for some vertex- and edge- but not arc-transitive group $G$, arise in this way. (The $G$-oriented graphs $\mathcal{G}(\Delta)$ may also be regarded as directed graphs. However, we choose to view them as undirected graphs with an orientation induced by the group, both because this is the viewpoint taken in the literature mentioned below, and also since a given undirected graph may admit several interesting orientations corresponding to different groups, see for example, [2].) Since the group $G$ acts transitively on vertices, all connected components of $\mathcal{G}(\Delta)$ are isomorphic, and the action induced by $G$ on each component is vertex-transitive, and edge-transitive, and preserves the $G$-invariant orientation. Thus we restrict attention to connected $G$-oriented graphs $\mathcal{G}(\Delta)$. Let $\mathcal{O G}(m)$ 
denote the family of pairs $(\mathcal{G}(\Delta), G)$, where $\Delta$ is a $G$-orbit on ordered pairs for a transitive permutation group $G$, and $\mathcal{G}(\Delta)$ is connected and $G$-oriented of valency $m$.

Marušič [24, p.221] described progress up to 1998 on studying $\mathcal{O G}(4)$ as 'thrilling'. One reason is undoubtedly a link to the study of maps on Riemann surfaces: research on this topic is led by Marušič and Nedela, and we sketch details of this link in Subsection 2.2. Another reason is the fascinating internal structure discovered by Marušič connected with so-called alternating cycles, which we discuss in Subsection 2.1. The latter study leads under certain conditions to a quotient of a pair $(\Gamma, G) \in \mathcal{O G}(4)$ which still lies in $\mathcal{O G}(4)$. However it is not clear how to describe the pairs $(\Gamma, G)$ for which this procedure gives no 'reduction'.

We present here a new framework for studying the family $\mathcal{O G}(4)$ based on a theory of normal graph quotients, and the identification of a sub-family of basic members of $\mathcal{O G}(4)$ with additional symmetry properties. This gives the potential of applying the theory of finite quasiprimitive permutation groups, and thereby exploiting the finite simple group classification to study these graphs. This has led us to new insights and new constructions of graphs in this family, to our knowledge not seen before in the literature. Our approach also provides a new framework for analysing known families of graphs in $\mathcal{O G}(4)$ (see below).

\section{Normal quotients.}

For a $G$-oriented graph $\Gamma$ with vertex set $X$, and a normal subgroup $N$ of $G$, we define the $G$-normal quotient $\Gamma_{N}$ as follows: the vertex set is the set of $N$-orbits in $X$, and a pair $\{B, C\}$ of distinct $N$-orbits forms an edge of $\Gamma_{N}$ if and only if there is at least one edge $\{x, y\}$ of $\Gamma$ with $x \in B$ and $y \in C$. Note that $\Gamma_{N}$ is defined as a graph with no specified orientation on its edges. Various degeneracies may occur when forming such quotients. For example, $\Gamma_{N}$ may consist of a single vertex if $N$ is vertex-transitive, and more generally, the valency of $\Gamma_{N}$ may be a proper divisor of the valency of $\Gamma$. The graph $\Gamma$ is called a $G$-normal $\ell$-multicover of $\Gamma_{N}$ if, for each edge $\{B, C\}$ of $\Gamma_{N}$, each vertex of $B$ is joined by an edge to exactly $\ell$ vertices of $C$ (or equivalently, each vertex of $C$ is joined by an edge to exactly $\ell$ vertices of $B$ ). In the case $\ell=1$ we usually say simply that $\Gamma$ is a $G$-normal cover of $\Gamma_{N}$. Our first result is an analogue for $G$-oriented graphs of the reduction theorem [13, Theorem 1.1] of Gardiner and the fourth author for arc-transitive graphs of valency 4. (A permutation group is semiregular if the only element fixing any point is the identity.)

Theorem 1.1. Let $(\Gamma, G) \in \mathcal{O G}(4)$ with vertex set $X$, and let $N$ be a normal subgroup of $G$. Then $G$ induces a permutation group $\bar{G}$ on the set of $N$-orbits in $X$, and either (i) $\left(\Gamma_{N}, \bar{G}\right)$ is also in $\mathcal{O G}(4), \Gamma$ is a $G$-normal cover of $\Gamma_{N}, N$ is semiregular on vertices, and $\bar{G}=G / N$; or (ii) $\left(\Gamma_{N}, \bar{G}\right)$ is degenerate as in one of the lines of Table 2 .

In Table 2, the graphs $K_{r}, C_{r}$ denote a complete graph and a cycle on $r$ vertices, respectively. Moreover, the cycle $\Gamma_{N}=C_{r}$ is $\bar{G}$-oriented if $\bar{G}=Z_{r}$, and $\bar{G}$-arc-transitive if $\bar{G}=D_{2 r}$; and we say that the cycle is oriented or unoriented, respectively. To set 


\begin{tabular}{cc|l}
\hline & & \\
$\Gamma_{N}$ & $\bar{G}$ & Comments \\
\hline$K_{1}$ & 1 & $N$ is vertex-transitive \\
$K_{2}$ & $Z_{2}$ & $\Gamma$ is bipartite, $N$-orbits form the bipartition \\
$C_{r}$ & $D_{2 r}$ & $r \geqslant 3, \Gamma$ is a $G$-normal 2-multicover of $\Gamma_{N}$ \\
$C_{r}$ & $Z_{r}$ & $r \geqslant 3, \Gamma$ is a $G$-normal 2-multicover of $\Gamma_{N}$ \\
\hline
\end{tabular}

Table 2: Degenerate cases for Theorem 1.1(ii)

this theorem in a broader context we analyse the possibilities for $G$-normal quotients of $G$-oriented graphs of arbitrary valency in Proposition 3.1 and derive Theorem 1.1 from this result. Theorem 1.1 shows that the family $\mathcal{O G}(4)$ is not closed under forming normal quotients as some of the degenerate cases in Table 2 may arise. We call the pairs $\left(\Gamma_{N}, \bar{G}\right)$ occurring in Table 2 degenerate pairs. Clearly the first line with $\Gamma_{N}=K_{1}$ occurs as a quotient for all $(\Gamma, G) \in \mathcal{O G}(4)$ (take $N=G$ ), and the second line occurs whenever the graph $\Gamma$ is bipartite (take $N$ the index 2 subgroup of $G$ stabilising the two parts of the bipartition). The other two lines of Table 2 can also occur (see for instance, Example 3.4 for line 4 , and see $[1,2]$ for more examples).

We call a pair $(\Gamma, G) \in \mathcal{O G}(4)$ basic if all of its $G$-normal quotients, relative to nontrivial normal subgroups of $G$, are degenerate pairs. Every $(\Gamma, G) \in \mathcal{O G}(4)$ has at least one basic $G$-normal quotient $\left(\Gamma_{N}, \bar{G}\right) \in \mathcal{O G}(4)$ (Lemma 3.3), and our aim is to explore the possible kinds of basic pairs. It is helpful to subdivide them broadly as described in Table 1, since each type gives additional information about the group action. If the only normal quotients are as in line 1 of Table 2, then all nontrivial normal subgroups of $G$ are transitive on the vertex set $X$; such groups $G$ are called quasiprimitive (Table 1, line 1). Similarly if the only normal quotients are as in lines 1 or 2 of Table 2 and line 2 does occur, then every nontrivial normal subgroup of $G$ has at most two orbits in $X$, and at least one normal subgroup has two orbits; such a group $G$ is called biquasiprimitive (Table 1, line 2). For all other basic pairs there is at least one normal quotient as in line 3 or 4 of Table 2, and the group $G$ has a dihedral or cyclic quotient, respectively (Table 1 , line 3).

Framework for studying $\mathcal{O G}(4)$. Theorem 1.1 and the remarks above suggest a new framework for studying oriented graph-group pairs in $\mathcal{O G}(4)$, consisting of the following general steps (see Table 1).

1. Develop a theory to describe the quasiprimitive basic pairs in $\mathcal{O G}(4)$.

2. Develop a theory to describe the biquasiprimitive basic pairs in $\mathcal{O G}(4)$.

3. Develop a theory to describe the basic pairs in $\mathcal{O G}(4)$ of cycle type.

4. Develop a theory to describe the $G$-normal covers $(X, G) \in \mathcal{O G}(4)$ of basic pairs of each of these three types. This theory should, for example, be powerful enough to 
describe, for a given basic pair $(Y, H) \in \mathcal{O G}(4)$, all pairs $(X, G) \in \mathcal{O G}(4)$ such that $X$ is a $G$-normal cover of $(Y, H)$.

5. Apply this theory: for a given pair $(X, G) \in \mathcal{O G}(4)$, determine whether or not $(X, G)$ is basic. If it is non-basic, then find a basic $G$-normal quotient of it in $\mathcal{O G}(4)$ (or all of its basic $G$-normal quotients).

Each of the steps in this framework requires delicate analysis. Substantial progress on completing the framework will provide a global structural view of the family $\mathcal{O G}(4)$. Regarding Step (4), there is a well-developed theory that will determine the normal covers $(X, G)$ of certain special types, for example, the normal quotients $Y=X_{N}$ modulo elementary abelian normal subgroups $N$ of $G$. The techniques used range from voltage assignments, representation theory, to studying universal covering groups, see recent expositions of the general theory (with good discussions of the literature) in $[9,23]$, and applications to $\mathcal{O G}(4)$ in $[10,33]$. A complete determination of all normal covers is probably not feasible.

In this paper we address Step (1) with short comments about Steps (2) and (3). We make only a few brief comments on basic pairs of cycle type, as these pairs will be the theme of further work by the authors in $[1,2]$. There are many infinite families of such graphs and we exhibit one such family in Example 3.4. A structure theorem is available to study quasiprimitive permutations groups in [38] analogous to the O'Nan-Scott theorem for studying finite primitive permutation groups. Here we apply this theory to determine the possible types of quasiprimitive groups $G$ that can arise for $(\Gamma, G) \in \mathcal{O G}(4)$, that is, $(\Gamma, G)$ is a quasiprimitive basic pair (Step 1 of the framework). It would be interesting to study biquasiprimitive basic pairs in a simlar way. A group theoretic tool for this is available in [40], but is far less detailed than the quasiprimitive analogue and is more difficult to apply.

Problem 1.2. Describe the biquasiprimitive basic graph-group pairs in $\mathcal{O G}(4)$.

Theorem 1.3. Suppose that $(\Gamma, G) \in \mathcal{O G}(4)$ is basic of quasiprimitive type. Then $G$ has a unique minimal normal subgroup $N=T^{k}$ for some finite nonabelian simple group $T$, $k \leqslant 2$, and one of the following holds.

(a) $k=1$, and there are many known examples; or

(b) $k=2, G=N \cdot 2$, and $\Gamma$ is a Cayley graph for $N$ as in Construction 4.7; or

(c) $k=2, N$ is not regular, and there are many known examples.

Remark 1.4. (i) As commentary on this result we note that Li, Lu and Marušič [20, Theorem 1.4] showed in 2004 that there are no vertex-primitive graph-group pairs in $\mathcal{O} \mathcal{G}(4)$. This confirms the suitability of normal quotient reduction to quasiprimitive actions as being the appropriate group theoretic reduction in the non-bipartite case.

(ii) An infinite family of examples for Theorem 1.3(a) with $T$ an alternating group $\operatorname{Alt}(n)$ was given by Marušič in [26]. In these examples $T$ is the full automorphism 
group and the vertex stabilisers are elementary abelian of unboundedly large order as $n$ grows. In Construction 5.3 we give a similar construction using the groups $\operatorname{Sym}(n)$, which also have unbounded vertex stabilisers. Our construction is slightly simpler than that in [26] since we do not have the restriction that $\operatorname{Sym}(n)$ is the full automorphism group. In addition we provide two general constructions, namely Construction 4.4 when $T$ is regular and Construction 5.1 when $T$ is not regular. Both constructions rely on certain 2-generation properties of the simple group $T$, and a small concrete example is given of each construction.

(iii) A general construction method is given in Construction 5.5 for examples satisfying Theorem 1.3(c), and a small concrete example is given. This construction also depends on certain 2-generation properties of nonabelian simple groups.

(iv) Theorem 1.3 shows that exactly three of the eight types of quasiprimitive groups $G$ arise in basic pairs $(\Gamma, G) \in \mathcal{O G}(4)$. These types are sometimes called As, Tw, PA for cases (a)-(c) respectively.

\section{Brief comments on edge-transitive oriented graphs}

If the full automorphism group of a finite $G$-oriented vertex-transitive, edge-transitive graph $\mathcal{G}(\Delta)$ preserves the orientation (and so does not act arc-transitively), then the graph $\mathcal{G}(\Delta)$ is called $\frac{1}{2}$-transitive or half-arc-transitive. Also, a graph admitting $G$ as a vertex-transitive and edge-transitive, but not arc-transitive, group of automorphisms is sometimes referred to in the literature as $\left(G, \frac{1}{2}\right)$-transitive. For example, an $n$-cycle $C_{n}$, relative to the cyclic group $G=Z_{n}$, is $G$-oriented, vertex-transitive and edge-transitive, and hence is $\left(G, \frac{1}{2}\right)$-transitive. However $C_{n}$ is not $\frac{1}{2}$-transitive since its full automorphism group $D_{2 n}$ is arc-transitive. In 1966, Tutte [56] asked whether any $\frac{1}{2}$-transitive graphs exist. As any connected graph of valency 2 is a cycle, it follows that all $\frac{1}{2}$-transitive graphs must necessarily have even valency at least 4. In 1970 Bouwer [5, Proposition 2] answered Tutte's question affirmatively by constructing, for each $k \geqslant 2$, a $\frac{1}{2}$-transitive graph of valency $2 k$ of order $6 \cdot 9^{k-1}$ (that is to say, having $6 \cdot 9^{k-1}$ vertices).

For each even integer $m \geqslant 2$, let $\mathcal{O G}(m)$ denote the family of all pairs $(\Gamma, G)$ such that $\Gamma$ is a connected $\left(G, \frac{1}{2}\right)$-transitive graph of valency $m$. The work of Bouwer shows that each $\mathcal{O G}(m) \neq \emptyset$, and we set $\mathcal{O G}:=\cup_{m} \mathcal{O G}(m)$. In fact, it is not difficult to construct pairs $(\Gamma, G)$ in $\mathcal{O G}(m)$ for arbitrary $m$ using a lexicographic product construction. In the decades since Bouwer's work these graphs have been well-studied. The major pioneer in this work is Dragan Marušič and in 1998 Marušič published an excellent survey [24] of results and open problems up to that time. Summaries of more recent work are available in $[19,30]$, and new advances appear regularly, such as the theory of alternets developed in $[18,57]$.

As Marušič remarked in [24], research on graph-group pairs in $\mathcal{O G}$ took three main directions: (i) the search for, and study of, pairs $(\Gamma, G)$ in $\mathcal{O G}$ with $G$ primitive on vertices; (ii) classification of pairs $(\Gamma, \operatorname{Aut}(\Gamma)) \in \mathcal{O G}$ of certain specified orders, for example, the order (number of vertices) being twice a prime, or a product of two primes, (or four times a prime [19]), etc; and (iii) an intensive study of the family $\mathcal{O G}(4)$. His paper [24] gives 
details of work in each of these three directions. We make a few brief comments here on the work of Marušič and others related to pairs $(\Gamma, G) \in \mathcal{O G}(4)$. In particular we discuss a different kind of quotienting operation for $\mathcal{O G}(4)$ (Section 2.1), the way such pairs arise from regular maps on surfaces (Section 2.2), and information on group structure making classification possible for orders up to 1,000 (Section 2.3),

\subsection{The alternating cycles of Marušič}

An alternating cycle in $\Gamma$, where $(\Gamma, G) \in \mathcal{O G}(4)$, is a cycle such that each pair of consecutive edges is oriented in opposite directions. Marušič [25, Proposition 2.4] showed that the alternating cycles all have the same even length and they partition the edge set of $\Gamma$. It is possible that there are only two alternating cycles and in this case Marušic proved that $\Gamma$ belongs to an explicitly described family of circulant graphs [25, Proposition 2.4]. If $\Gamma$ contains more than two alternating cycles, then the non-empty intersections of the vertex sets of distinct alternating cycles have a fixed size, called the attachment number of $(\Gamma, G)$, and form a system of blocks of imprimitivity for $G$. Moreover, the corresponding quotient graph is also a member of $\mathcal{O G}(4)$ relative to the induced $G$-action [29, Theorem 1.1 and Theorem 3.6]. It is possible that the attachment number is 1 , and in this case the alternating cycles are said to be loosely attached, the quotient is just the graph $\Gamma$, and no reduction is achieved. However, for any positive integer $k$, there are infinitely many examples of pairs $(\Gamma, G) \in \mathcal{O G}(4)$ with attachment number $k$ [31]. Also if the attachment number is at least 3 , then a vertex stabiliser $G_{x}$ has size 2 [29, Lemma 3.5]. The attachment number is at most half the length of an alternating cycle. When this maximum is attained the cycles are said to be $G$-tightly attached, and all pairs $(\Gamma, G)$ in this case have been classified, in [25, Theorem 3.4] and [29, Lemma 4.1 and Theorem 4.5] when the attachment number is odd and even, respectively. This classification has been 'simplified and sharpened' by Wilson [57, Section 8], and all $\frac{1}{2}$-transitive examples identified by Šparl [48].

The possibility of understanding the internal structure of graph-group pairs in $\mathcal{O G}(\mathrm{m})$ for larger values of $m$, by identifying smaller quotients in the family is tantalising, and as far as we know has not been explored for general valencies $m$. Very recent work of Hujdurović [18] studies vertex subsets of graphs in $\mathcal{O G}(m)$ called alternets, which are analogues for general $m$ of alternating cycles and were introduced in [57, Section 4]. They are equivalence classes of the so-called reachability relation introduced in [7] for infinite arc-transitive digraphs. The paper [18] focuses on oriented graphs with a small number of alternets.

\subsection{Regular maps and their medial graphs}

Cellular decompositions of surfaces are called maps, and a common way to construct maps is by embedding a graph into a surface. For a map $M$ given by embedding a graph $\Sigma$ into some surface, the map group Aut $M$ is the subgroup of automorphisms preserving the surface, and $M$ is called regular if Aut $M$ acts transitively (and therefore regularly) on the set of flags of $M$ (mutually incident triples consisting of a vertex, edge, 
and face). Regular maps are extensively studied in various branches of mathematics, including combinatorics, Riemann surfaces and group theory [12, 16, 49, 53] going back to work of Tutte and Dyck, with many recent papers inspired by the ground-breaking work of Jones and Singerman [16].

Some pairs $(\Gamma, G) \in \mathcal{O G}(4)$ arise as medial graphs of regular maps, and the work of Nedela and Marusic [27] established medial graphs/maps as a fundamental tool for studying regular maps on surfaces. The medial graph of a map $M$ (embedded into the same surface as $M$ ) is described as follows, see [27, page 346] or [55]: subdivide each edge of $M$ with one new vertex. If $e$ and $f$ are two consecutive edges in a boundary walk of a face $F$ of $M$, then join the two corresponding new vertices by a new edge in $F$. Finally remove from the surface all the original vertices together with incident (old) edges. We obtain a 4-valent graph $\operatorname{Med}(M)$ embedded into the surface, called the medial graph of $M$. By its construction, it is embedded in the same surface as the original map $M$, and this embedded graph is called the medial map of $M$. Marušič and Nedela [27, Proposition 2.1] showed that $(\operatorname{Med}(M), G) \in \mathcal{O G}(4)$, where $M$ is a regular map and $G$ is the map group, and moreover vertex stabilisers $G_{x}$ have order 2. They also proved a converse in $\left[27\right.$, Proposition 2.2], namely that each $(\Gamma, G) \in \mathcal{O G}(4)$ with vertex stabilisers $G_{x}$ of order 2 arises as a medial map of some regular map $M$ with automorphism group $G$ such that $\operatorname{Med}(M) \cong \Gamma$; and $M$ is determined up to duality and reflection. They also show [27, Theorem 4.1(4)] that for the special class of 'negatively self-dual' regular maps $M$, the medial graph $\operatorname{Med}(M)$ admits a group $G$ which is twice as large as the map group of $M$, giving a second pair $(\operatorname{Med}(M), G) \in \mathcal{O G}(4)$, this time with vertex stabilisers $Z_{2}^{2}$.

\section{$2.3 \quad$ Vertex stabilisers}

As mentioned above, pairs $(\Gamma, G) \in \mathcal{O G}(4)$ with attachment number at least 3 have vertex stabilisers of order 2, and the sizes of vertex stabilisers for graph-group pairs in $\mathcal{O G}(4)$ associated with medial graphs can be at most 4, while the vertex stabilisers of arbitrary pairs in $\mathcal{O G}(4)$ can be unboundedly large, even with $G$ a finite alternating group [26, Theorem 1.1]. In general the vertex stabilisers are 2-groups of nilpotency class at most 2 , and they form the family of concentric 2-groups, studied by Marušič and Nedela, see [28, Sections 5-7], and first discovered by Glauberman [14] when attacking a rather different problem. (In fact, roughly speaking, Glauberman in [14] investigates groups $G$ containing a finite $p$-subgroup $P$ and an element $g$ such that $G=\left\langle P, P^{g}\right\rangle$ with $\left|P: P \cap P^{g}\right|=p$. By taking $G$ to be a $\frac{1}{2}$-transitive subgroup of automorphisms of a 4 -valent graph, $P$ to be a vertex stabiliser $G_{x}$, and $g$ an element of $G$ mapping $x$ to one of its neighbours, we see that $G, P, g$ satisfy the hypotheses considered by Glauberman. Hence some of the results of Marušič and Nedela follow from the work of Glauberman.) The smallest nonabelian concentric 2-group is the dihedral group $D_{8}$ of order 8 , and the first construction of a $\frac{1}{2}$-transitive graph with vertex stabilisers $D_{8}$ was given by Conder and Marušič in [8]. Their graph has 10,752 vertices, and it was recently proved by Potočnik and Požar [33] that there are exactly two $\frac{1}{2}$-transitive graphs of this order and no such graphs with fewer vertices. Links between the stabiliser orders $\left|G_{x}\right|$ and the graph structure, have been studied in $[27,36,47]$. In particular, the information in [47] was sufficiently powerful to 
enable Potočnik, Spiga and Verret [35] to classify all members of $\mathcal{O G}(4)$ with up to 1,000 vertices. It would be worth exploring realisations of concentric groups as vertex stabilisers in basic pairs $(\Gamma, G) \in \mathcal{O} \mathcal{G}(4)$.

\section{$3 \quad G$-oriented graphs and their normal quotients}

For fundamental graph theoretic concepts please refer to the book [15].

\subsection{G-oriented edge transitive graphs}

Suppose that $G \leqslant \operatorname{Sym}(X)$ is a transitive permutation group on $X$, and that $\Delta \subset X \times X$ is a non-diagonal $G$-orbit such that the associated graph $\mathcal{G}(\Delta)$ is $G$-oriented and connected. As mentioned in the introduction $\mathcal{G}(\Delta)$ admits $G$ as a vertex-transitive and edge-transitive group of automorphisms, and each edge $\{x, y\}$ is oriented from $x$ to $y$ if and only if $(x, y) \in \Delta$. Let $\mathcal{O G}$ denote the set of all such graph-group pairs $(\mathcal{G}(\Delta), G)$.

The set $\Delta^{*}:=\{(y, x) \mid(x, y) \in \Delta\}$ is also a $G$-orbit and is disjoint from $\Delta$ since $\mathcal{G}(\Delta)$ is $G$-oriented. Moreover the graph $\mathcal{G}\left(\Delta^{*}\right)$ is $G$-oriented and has the same underlying undirected graph as $\mathcal{G}(\Delta)$ with each edge oriented in the opposite direction. The two $G$-oriented graphs $\mathcal{G}(\Delta)$ and $\mathcal{G}\left(\Delta^{*}\right)$ may, or may not, be isomorphic as oriented graphs: they are ismorphic if and only if there is an automorphism $h$ of the underlying graph which maps some vertex-pair in $\Delta$ to a pair in $\Delta^{*}$. Necessarily $h \notin G$ since $G$ fixes $\Delta$ setwise, and such an automorphism exists if and only if $\mathcal{G}(\Delta)$ is arc-transitive relative to a group larger than $G$. Note that it is not always possible to choose the automorphism $h$ to interchange the $G$-orbits $\Delta$ and $\Delta^{*}$, see [10, Section 6], where it is claimed that the smallest graph $\Gamma$ for which this is the case has 21 vertices. We checked the possibilities and confirmed that there exists a unique 4 -valent, arc-transitive graph $\Gamma$ with 21 vertices. It has automorphism group PGL $(2,7)$ and dihedral vertex stabilisers of order 16 (not of order 8 as claimed in [10]). There is a subgroup $G$ (a Frobenius group of order 42) such that $(\Gamma, G) \in \mathcal{O G}(4)$, and no automorphism of $\Gamma$ interchanges the two $G$-orbits on arcs.

It follows from $[17,43]$ that every graph or oriented graph admitting $G$ as an edgetransitive and vertex-transitive group of automorphisms arises as $\mathcal{G}(\Delta)$ for some nondiagonal $G$-orbit $\Delta$ on ordered vertex-pairs. We note also that, by a result of Sims $[45$, Proposition 3.1], a $G$-oriented graph $\mathcal{G}(\Delta)$ is strongly connected, in the sense that for every pair of distinct vertices there is an oriented path from the first to the second, if and only if the underlying undirected graph is connected (each pair of vertices is joined by a path in the graph with no restriction on the orientation of the edges in the path).

For a positive integer $k$, let $\mathcal{A G}(k)$ denote the set of graph-group pairs $(\Sigma, H)$ such that $\Sigma$ is a connected undirected graph of valency $k$, and $H \leqslant \operatorname{Aut}(\Sigma)$ acts arc-transitively. Also let $\mathcal{A G}:=\cup_{k \geqslant 1} \mathcal{A G}(k)$, and let $\mathcal{O G}:=\cup_{m \text { even }} \mathcal{O G}(m)$, where as in the introduction $\mathcal{O G}(m)$ is the set of pairs $(\Gamma, G)$ such that $\Gamma$ is a connected $G$-oriented graph of valency $m$, and $G \leqslant \operatorname{Aut}(\Gamma)$ acts transitively on vertices and edges. 


\subsection{Normal quotients of $G$-oriented graphs}

Let $(\Gamma, G) \in \mathcal{O G}$ with vertex set $X$ and of even valency $m \geqslant 4$, so $\Gamma$ is connected and $\Gamma=\mathcal{G}(\Delta)$ for some non-diagonal $G$-orbit $\Delta$ in $X \times X$ such that $\Delta \neq \Delta^{*}$. Let $N$ be a normal subgroup of $G$, and recall the definition of the normal quotient graph $\Gamma_{N}$ given in the introduction. Normal quotients were studied in 1989 by the fourth author [37] focussing on the special subfamily of $\mathcal{O G}$ consisting of pairs $(\Gamma, G)$ such that $G$ is transitive on directed paths of length 2 in $\Gamma$ (see [37, Section 3], especially Lemma 3.2 and Theorem 3.3). Proposition 3.1 is a generalisation of those results.

Since the $N$-orbits form a $G$-invariant partition of $X$ (see for example [39, Lemma 10.1]), and since $G$ is transitive on $X$ and on $\Delta$, it follows from the definitions of $\Gamma=\mathcal{G}(\Delta)$ and $\Gamma_{N}$ that $G$ induces a group $\bar{G}$ of automorphisms of $\Gamma_{N}$ which is transitive on both vertices and edges. One possibility is that $N$ has only one orbit on vertices (for example, if $N=G$ ) and then $\Gamma_{N}=K_{1}$ consists of a single vertex. In all other cases $\Gamma_{N}$ contains edges since $\Gamma$ is connected, and it is fairly easy to see that $\Gamma_{N}$ must itself be connected. It is possible that $\Gamma_{N}$ inherits an orientation from the $G$-orientation of $\Gamma$, namely if, for some (and hence every) edge $\{B, C\}$ of $\Gamma_{N}$, either all of the edges $\{x, y\}$ of $\Gamma$ with $x \in B$ and $y \in C$ are such that $(x, y) \in \Delta$, or all of these edges have $(x, y) \in \Delta^{*}$. In this case we prove in Proposition 3.1 that $\left(\Gamma_{N}, \bar{G}\right) \in \mathcal{O G}$. If $\Gamma_{N}$ does not have this property, then we show that every edge $\{B, C\}$ of $\Gamma_{N}$ has $\Gamma$-edges 'in both directions' between vertices of $B$ and $C$, and $\bar{G}$ is arc-transitive on $\Gamma_{N}$.

Proposition 3.1. Let $(\Gamma, G) \in \mathcal{O G}(m)$ with vertex set $X$ and $m \geqslant 4$, let $N$ be a normal subgroup of $G$. Then $\Gamma_{N}$ is connected, $G$ induces a permutation group $\bar{G}$ on the set of $N$-orbits in $X$, and either $\left(\Gamma_{N}, \bar{G}\right)=\left(K_{1}, 1\right)$, or one of the following holds.

(a) $\left(\Gamma_{N}, \bar{G}\right) \in \mathcal{O G}(k)$ and $\Gamma$ is a $G$-normal $(m / k)$-multicover of $\Gamma_{N}$, for some even divisor $k$ of $m$; or

(b) $\left(\Gamma_{N}, \bar{G}\right) \in \mathcal{A G}(k)$ and $\Gamma$ is a $G$-normal $(m / k)$-multicover of $\Gamma_{N}$, for some divisor $k$ of $m$ with $m / k$ even (so $1 \leqslant k \leqslant m / 2$ ).

Remark 3.2. Consider Proposition 3.1 case (b) with $k=1$. Here, for $x$ in an $N$-orbit $B$, say, all $m$ of the edges incident with $x$ have their other end point in a single second $N$-orbit, $C$ say, and by connectivity we find that there are just two $N$-orbits, so $\Gamma$ is bipartite, the $N$-orbits form the bipartition, and $\left(\Gamma_{N}, \bar{G}\right)=\left(K_{2}, Z_{2}\right)$.

Proof. We use the notation and discussion given before the statement, so $\Gamma=\mathcal{G}(\Delta)$ for some $G$-orbit $\Delta \neq \Delta^{*}$ in $X \times X$, the quotient $\Gamma_{N}$ is connected, and the induced group $\bar{G}$ is transitive on the vertices and edges of $\Gamma_{N}$. Suppose that $\Gamma_{N} \neq K_{1}$ so there are at least two $N$-orbits. Since $\Gamma_{N}$ is connected, $\Gamma_{N}$ has at least one edge $\{B, C\}$ and by definition there are vertices $x \in B, y \in C$, which form an edge of $\Gamma$. Without loss of generality $(x, y) \in \Delta$. Let $\Delta(x)=\{z \mid(x, z) \in \Delta\}$, and let $\ell_{C}(x)=|\Delta(x) \cap C|$. Since $y \in \Delta(x) \cap C$, we have $\ell_{C}(x)>0$. Moreover, since $G$ is transitive on $\Delta$ it follows that $G_{x}$ is transitive on $\Delta(x)$, and hence $\ell_{C^{\prime}}(x)=\ell_{C}(x)=\ell$, say, for all $N$-orbits $C^{\prime}$ that meet $\Delta(x)$ nontrivially. Thus $\ell$ divides $|\Delta(x)|$, and as the valency $m$ of $\Gamma$ is equal to $|\Delta(x)|+\left|\Delta^{*}(x)\right|=2|\Delta(x)|$, 
we have $2 \ell \mid m$. Further, since $N$ is transitive on $B$ and fixes $C$ setwise, it follows that $\ell=\ell_{C}(x)$ is independent of the vertex $x$ of $B$ and hence the number of pairs from $B \times C$ lying in $\Delta$ is equal to $|B| \ell=|C| \ell$. Since also $N$ is transitive on $C$ and fixes $B$ setwise, we find that $\ell=\left|\Delta^{*}(z) \cap B\right|$ for each $z \in C$.

Suppose first that there are no edges of $\Gamma$ between $B$ and $C$ that are oriented 'from $C$ to $B^{\prime}$, that is to say, $\Delta$ contains no pairs from $C \times B$. Since $\bar{G}$ is transitive on the edges of $\Gamma_{N}$, this is true for all $\Gamma_{N}$-edges, and hence $\Gamma_{N}$ admits a $\bar{G}$-invariant orientation from the $G$-orientation of $\Gamma$, that is $\left(\Gamma_{N}, \bar{G}\right) \in \mathcal{O G}$. Moreover there are exactly $|B| \ell$ edges of $\Gamma$ joining vertices in $B$ to vertices in $C$, and so $\Gamma$ is a $G$-normal $\ell$-multicover of $\Gamma_{N}$. Finally, since there are exactly $|B| m$ edges of $\Gamma$ with one end in $B$, it follows that the valency $k$ of $\Gamma_{N}$ is $k=|B| m /(|B| \ell)=m / \ell$, which is even since $2 \ell \mid m$. Thus (a) holds.

Now suppose that there is also at least one $\Gamma$-edge from $C$ to $B$, that is, there is a pair $(c, b) \in \Delta$ with $c \in C, b \in B$. Since $G$ is transitive on $\Delta$, some element $g \in G$ maps $(x, y)$ to $(c, b)$, and hence $g$ interchanges $B$ and $C$. This means that $\bar{G}$ acts arc-transitively on $\Gamma_{N}$, so $\left(\Gamma_{N}, \bar{G}\right) \in \mathcal{A G}$. Further, $g$ maps the set $\Delta \cap(B \times C)$ to $\Delta \cap(C \times B)$, and it follows that each vertex $x$ of $B$ is joined in $\Gamma$ to $\ell$ vertices of $\Delta^{*}(x) \cap C$. Thus there are $2 \ell|B|$ edges of $\Gamma$ between vertices of $B$ and $C$ (namely $\ell|B|$ in each orientation), so $\Gamma$ is

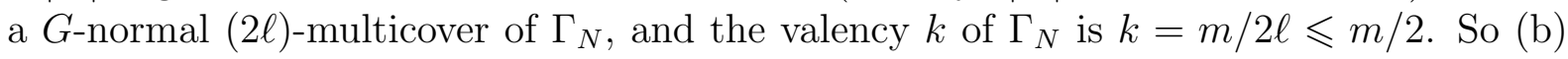
holds.

We derive Theorem 1.1 from Proposition 3.1.

Proof of Theorem $\mathbf{1 . 1}$ If $N$ is transitive on $X$ then $\Gamma_{N}=K_{1}, \bar{G}=1$, and line 1 of Table 2 holds. So suppose that $N$ is intransitive. Then $\Gamma_{N} \neq K_{1}$ and so (a) or (b) of Proposition 3.1 holds with $m=4$. In particular $\Gamma_{N}$ is connected. Suppose first that case (a) holds with $k=4$, so that $\Gamma$ is a $G$-normal cover of $\Gamma_{N}$. Since $N$ fixes each vertex of $\Gamma_{N}$ setwise, $\bar{G}=G / K$ for some normal subgroup $K$ of $G$ containing $N$. Moreover the $K$-orbits are the same as the $N$-orbits. For each vertex $x$, the four vertices adjacent to $x$ in $\Gamma$ lie in four distinct $N$-orbits, and hence the stabiliser $K_{x}$ fixes pointwise the vertices adjacent to $x$, and by the connectivity of $\Gamma$ it follows that $K_{x}=1$. Thus $K$ is semiregular on vertices, since this holds for all vertices $x$. Also $|K|$ is equal to the size of the $N$-orbit containing $x$, and this is a divisor of $|N|$. Hence $K=N$ and thus $\bar{G}=G / N$, and Theorem 1.1 (i) holds.

If case (a) of Proposition 3.1 holds with $k=2$, then $\Gamma$ is a $G$-normal 2-multicover of $\Gamma_{N}$, and as $\Gamma_{N}$ is an oriented connected 2-valent graph it follows that $\Gamma_{N}=\mathrm{C}_{r}$ and $\bar{G}=Z_{r}$, for some $r \geqslant 3$, as in line 4 of Table 2 .

Suppose now that case (b) of Proposition 3.1 holds. If $k=1$ then, as discussed in Remark 3.2, $\Gamma_{N}=K_{2}, \bar{G}=Z_{2}, \Gamma$ is bipartite, and the $N$-orbits form the bipartition, as in line 2 of Table 2 . If $k>1$ then the only possibility is $k=2$, and then $\Gamma$ is a $G$-normal 2-multicover of $\Gamma_{N}$, and $\Gamma_{N}$ is a connected $\bar{G}$-arc-transitive graph of valency 2 , so $\Gamma_{N}=C_{r}$ and $\bar{G}=D_{2 r}$, for some $r \geqslant 3$, as in line 3 of Table 2 . 


\subsection{Basic pairs in $\mathcal{O G}(4)$}

It is not difficult to see that every non-basic $(\Gamma, G) \in \mathcal{O G}(4)$ has at least one basic normal quotient.

Lemma 3.3. For each non-basic $(\Gamma, G) \in \mathcal{O G}(4), \Gamma$ is a $G$-normal cover of at least one quotient $\Gamma_{N}$, with $\left(\Gamma_{N}, G / N\right) \in \mathcal{O G}(4)$ basic.

Proof. By definition of a basic pair, since $(\Gamma, G) \in \mathcal{O G}(4)$ is non-basic, it follows from Theorem 1.1 that there is a nontrivial normal subgroup $N$ of $G$ such that the quotient $\left(\Gamma_{N}, \bar{G}\right) \in \mathcal{O G}(4)$ and $\Gamma$ is a $G$-normal cover of $\Gamma_{N}$, where $\bar{G}=G / N$.

If $\left(\Gamma_{N}, \bar{G}\right) \in \mathcal{O G}(4)$ is basic there is nothing further to prove, so suppose that this is not the case. Then, as in the previous paragraph, there is a nontrivial normal subgroup $\bar{M}$ of $\bar{G}$ such that the quotient $\left(\left(\Gamma_{N}\right)_{\bar{M}}, \bar{G} / \bar{M}\right) \in \mathcal{O G}(4)$ and $\Gamma_{N}$ is a $\bar{G}$-normal cover of $\left(\Gamma_{N}\right)_{\bar{M}}$.

Each such subgroup $\bar{M}$ is of the form $M / N$ for a (unique) normal subgroup $M$ of $G$ properly containing $N$. Moreover, it follows from the definitions that $\left(\Gamma_{N}\right)_{\bar{M}} \cong \Gamma_{M}$ and the group $\bar{G} / \bar{M}=(G / N) /(M / N) \cong G / M$ so we have a larger normal subgroup $M$ of $G$ and a smaller normal quotient $\left(\Gamma_{M}, G / M\right) \in \mathcal{O G}(4)$, and $\Gamma$ is a $G$-normal quotient of $\Gamma_{M}$.

Since the group $G$ is finite this process can be applied a finite number of times, yielding a strictly increasing chain of normal subgroups of $G$, namely $1<N_{1}<N_{2}<\cdots<$ $N_{s}$, and a corresponding sequence of $G$-normal quotients $\left(\Gamma_{N_{1}}, G / N_{1}\right),\left(\Gamma_{N_{2}}, G / N_{2}\right), \ldots$, $\left(\Gamma_{N_{s}}, G / N_{s}\right)$ in $\mathcal{O G}(4)$ such that the final pair $\left(\Gamma_{N_{s}}, G / N_{s}\right)$ is basic, and $\Gamma$ is a $G$-normal cover of $\Gamma_{N_{s}}$.

As mentioned in Subsection 2.3, for each $(\Gamma, G) \in \mathcal{O G}(4)$, a vertex stabiliser $G_{x}$ is a nontrivial 2-group, and the possible orders $\left|G_{x}\right|$ for $(\Gamma, G) \in \mathcal{O G}(4)$ are unbounded, even in the case of basic graphs. This was established in [26] for the case where $G$ is an alternating group. We give here a simple example of basic cycle type, and we give further examples which are basic of quasiprimitive type in Construction 5.3.

Example 3.4. Let $r \geqslant 3$ and let $\Gamma$ be the graph with vertex set $X=\mathbb{Z}_{r} \times \mathbb{Z}_{2}$ and edges $\left\{(i, j),\left(i+1, j^{\prime}\right)\right\}$ for all $i \in \mathbb{Z}_{r}, j, j^{\prime} \in \mathbb{Z}_{2}$, that is, $\Gamma=C_{r}\left[2 . K_{1}\right]$, the lexicographic product of $C_{r}$ and $2 . K_{1}$. We orient each edge so that $(i, j) \rightarrow\left(i+1, j^{\prime}\right)$. Let $G=Z_{2} \imath Z_{r}=$ $\left\{\left(\sigma_{1}, \ldots, \sigma_{r}\right) \tau^{\ell} \mid 0 \leqslant \ell<r\right.$ and each $\left.\sigma_{k} \in \mathbb{Z}_{2}\right\}$, where $\left(\sigma_{1}, \ldots, \sigma_{r}\right):(i, j) \mapsto\left(i, j+\sigma_{i}\right)$, and $\tau:(i, j) \mapsto(i+1, j)$.

Lemma 3.5. Let $r, \Gamma, G, X$ and the edge orientation be as in Example 3.4. Then $(\Gamma, G) \in$ $\mathcal{O G}(4)$, and for $x \in X, G_{x} \cong Z_{2}^{r-1}$. Moreover, each $(\Gamma, G)$ is basic of cycle type.

Proof. By definition the graph $\Gamma$ is a connected graph of valency 4 . It is straightforward to show that $G$ acts as a group of automorphisms of $\Gamma$ which preserves the orientation, and is transitive on both vertices and edges, so $(\Gamma, G) \in \mathcal{O G}(4)$. As $G$ is vertex-transitive, without loss of generality we choose $x=(0,0) \in X$. The stabiliser is $G_{x}=\left\{\left(0, \sigma_{1}, \ldots, \sigma_{r}\right) \mid\right.$ each $\left.\sigma_{k} \in \mathbb{Z}_{2}\right\} \cong Z_{2}^{r-1}$. 
First we consider the quotient $\Gamma_{B}$ modulo the 'base group'

$$
B=\left\{\left(\sigma_{1}, \ldots, \sigma_{r}\right) \mid \text { each } \sigma_{k} \in \mathbb{Z}_{2}\right\}=Z_{2}^{r} .
$$

This group is normal in $G$ and its orbits are the sets $Y_{i}:=\{(i, 0),(i, 1)\}$, for $i \in \mathbb{Z}_{r}$. From the definition of the edge orientation of $\Gamma$, it follows that $\Gamma_{B}$ is the cycle $C_{r}$, and is $G / B$-oriented.

Next consider a minimal normal subgroup $N$ of $G$. We claim that $N$ is contained in $B$. If this is not the case then $N \cap B=1$ and so $N \leqslant C_{G}(B)$. However $C_{G}(B)=B$ and we have a contradiction. Thus $N \leqslant B$. Since all $B$-orbits have size 2 , and since all $N$-orbits must have equal size (because $N$ is normal in $G$ ), it follows that $N$ and $B$ have the same orbits, and hence $\Gamma_{N}=\Gamma_{B}=C_{r}$.

Now let $M$ be an arbitrary normal subgroup of $G$. Then $M$ contains a minimal normal subgroup, and it follows from the previous paragraph that the $M$-orbits must be unions of $B$-orbits. Thus the quotient $\Gamma_{M}$ is isomorphic to a quotient of $\Gamma_{B}$ (possibly equal to $\left.\Gamma_{B}\right)$. Hence $\Gamma_{M}$ is a cycle, or possibly $K_{2}$ or $K_{1}$, and so $(\Gamma, G)$ is basic of cycle type.

\section{Cayley graphs in $\mathcal{O G}(4)$}

Many of the $\frac{1}{2}$-transitive graphs of valency four in the literature are Cayley graphs, and we need the following information about them for our analysis.

(i) For a group $N$ and inverse-closed subset $S$ of $N \backslash\left\{1_{N}\right\}$, the Cayley graph $\operatorname{Cay}(N, S)$ has vertex set $N$, and edges $\{x, y\}$ such that $x y^{-1} \in S$.

(ii) $\operatorname{Cay}(N, S)$ admits $N$ in its right multiplication action $(g: x \mapsto x g$ for $x, g \in N)$ as a subgroup of automorphisms that is regular on vertices (that is, $N$ is transitive, and only the identity fixes a point).

(iii) $\operatorname{Cay}(N, S)$ is connected if and only if $S$ generates $N$.

(iv) If $S=S_{0} \cup S_{0}^{-1}$ with $S_{0} \cap S_{0}^{-1}=\emptyset$, then for any pair $x, y$ of distinct vertices, at most one of $x y^{-1}$ and $y x^{-1}$ lies in $S_{0}$, and we may define an orientation on each edge $\{x, y\}$ by $x \rightarrow y$ if and only if $y x^{-1} \in S_{0}$.

Remark 4.1. To explain how Cayley graphs arise naturally in our investigation, we remark the following: if $G \leqslant \operatorname{Aut}(\Gamma)$ for a graph $\Gamma$ with vertex set $X$, and if $N$ is a normal subgroup of $G$ that is regular on $X$, then we may identify $X$ with $N$ and there exists $S \subset N$ such that $\Gamma=\operatorname{Cay}(N, S)$, and $G$ is a semidirect product $G=N \rtimes H$, where $N$ acts on $X=N$ by right multiplication, and $H \leqslant \operatorname{Aut}(N)$ acts naturally as automorphisms fixing $S$ setwise. Moreover, $H$ is the stabiliser in $G$ of the vertex $1_{N}$, and the only element of $H$ fixing $S$ pointwise is the identity, since $S$ generates $N$. See, for example, [6, Section 1.7]. 
Lemma 4.2. Let $(\Gamma, G) \in \mathcal{O G}(4)$, and let $N$ be a regular normal subgroup of $G$. Then $\Gamma=$ $\operatorname{Cay}(N, S)$, with $S=S_{0} \cup S_{0}^{-1}$ and $S_{0}=\{a, b\}$ such that $N=\langle S\rangle$ and $1_{N} \notin\left\{a^{2}, b^{2}, a b\right\}$. Also $G=N \rtimes H$, where $H \leqslant \operatorname{Aut}(N)$, $H$ leaves $S_{0}$ invariant, and $H \cong Z_{2}$ interchanges $a$ and $b$.

Proof. By Remark 4.1, since $N$ is a regular normal subgroup of $G$, we have $\Gamma=\operatorname{Cay}(N, S)$, and $G=N \rtimes H$, where $H$ is a subgroup of $\operatorname{Aut}(N)$ which leaves $S$ invariant, $H$ is the stabiliser of $1_{N}$, and $H$ acts faithfully on $S$. Also, since $\Gamma$ is connected, $N=\langle S\rangle$. Moreover, since $(\Gamma, G) \in \mathcal{O} \mathcal{G}(4)$, there are two distinct elements of $N$, say $a$ and $b$, such that $1_{N} \rightarrow a$ and $1_{N} \rightarrow b$. Let $S_{0}=\{a, b\}$. Then by the definition of adjacency we have $S_{0} \subset S$. The images of these oriented edges under the actions of $a^{-1}, b^{-1} \in N$ are $a^{-1} \rightarrow 1_{N}$ and $b^{-1} \rightarrow 1_{N}$, respectively. Thus also $S_{0}^{-1}=\left\{a^{-1}, b^{-1}\right\} \subset S$. Since $1_{N}$ has exactly two 'out-neighbours' and two 'in-neighbours', it follows that $S=S_{0} \cup S_{o}^{-1}$ and $S_{0} \cap S_{0}^{-1}=\emptyset$. In particular $a^{-1}, b^{-1} \notin S_{0}$ and hence $1_{N} \notin\left\{a^{2}, b^{2}, a b\right\}$. Since $G$ preserves the edge orientation and is edge-transitive, the stabiliser $H$ of $1_{N}$ must interchange $a$ and $b$, so $H \cong Z_{2}$.

In the case where $N$ is a regular minimal normal subgroup of $G$, the conditions of Lemma 4.2 are sufficient to exclude the possibility that $N$ is abelian (Lemma 4.3), and to allow us to construct examples with $N$ nonabelian (Constructions 4.4 and 4.7).

Lemma 4.3. If $(\Gamma, G) \in \mathcal{O G}(4)$ with $\Gamma=\operatorname{Cay}(N, S)$ for a minimal normal subgroup $N$ of $G$, then $N$ is not abelian.

Proof. Suppose that $N$ is an abelian minimal normal subgroup of $G$. Then $N=Z_{p}^{d}$ for some prime $p$ and integer $d \geqslant 1$. By Lemma 4.2, $S=S_{0} \cup S_{0}^{-1}, S_{0}=\{a, b\}$, and $G=N \rtimes H$ with $H=Z_{2}$ interchanging $a$ and $b$. This implies, since $N$ is abelian, that $H$ fixes $a b=b a$, and by Lemma $4.2, a b \neq 1$. Since $N$ is abelian the subgroup $\langle a b\rangle$ is therefore normal in $G$, and since $N$ is a minimal normal subgroup, this means that $N=\langle a b\rangle$, so $d=1$. In this case, however, the only subgroup $H$ of $\operatorname{Aut}(N)$ of order 2 inverts $N$, and hence $b=a^{-1}$, which is a contradiction.

Construction 4.4. Let $N$ be a nonabelian simple group and $a \in N, \sigma \in \operatorname{Aut}(N)$, such that $\sigma^{2}=1, S_{0}:=\left\{a, a^{\sigma}\right\}$ generates $N$, and let $S=S_{0} \cup S_{0}^{-1}$. Then for $\Gamma=\operatorname{Cay}(N, S)$ and $G=N \rtimes\langle\sigma\rangle$, we have $(\Gamma, G) \in \mathcal{O G}(4)$.

Lemma 4.5. Each $(\Gamma, G)$ in Construction 4.4 lies in $(\Gamma, G) \in \mathcal{O G}(4)$ and is basic of quasiprimitive type.

Proof. From our discussion above, $G \leqslant \operatorname{Aut}(\Gamma)$, and $G$ is transitive on both the vertex set $N$ and the edge set of $\Gamma$. It remains to prove that $S_{0} \cap S_{0}^{-1}=\emptyset$, since from this it will follow that $\Gamma$ is $G$-oriented of valency 4 . First we note that $a^{-1} \neq a$, since otherwise $S_{0}$ would consist of two involutions and so cannot generate a nonabelian simple group. If $a^{-1}=a^{\sigma}$, then $S_{0}$ generates a cyclic group. Thus $a^{-1} \notin S_{0}$ and similarly $\left(a^{\sigma}\right)^{-1} \notin S_{0}$. 
For an example of a graph with the properties of Lemma 4.5, take $N=\operatorname{Alt}(5)$, $a=(123)$, and $\sigma \in \operatorname{Aut}(N)=\operatorname{Sym}(5)$ the inner automorphism induced by conjugation by (14)(25). It would be interesting to have a good understanding of the family of Cayley graphs arising in Lemma 4.5. In particular generation results such as those in [22] should give some insights.

Problem 4.6. Determine all the Cayley graphs $\Gamma$ for nonabelian simple groups $T$ such that $(\Gamma, G) \in \mathcal{O G}(4)$ for some $G \leqslant \operatorname{Aut}(T)$.

Construction 4.7. Let $T$ be a nonabelian simple group, and let $\{a, b\}$ be a generating set for $T$ such that no automorphism of $T$ interchanges $a$ and $b$. Let $N=T \times T$, $S_{0}=\{(a, b),(b, a)\}, S=S_{0} \cup S_{0}^{-1}$, and $\Gamma=\operatorname{Cay}(N, S)$. Let $\tau \in \operatorname{Aut}(N)$ be the map $\tau:(x, y) \mapsto(y, x)$, and let $G=N \rtimes\langle\tau\rangle$.

All nonabelian simple groups have many generating pairs satisfying the requried conditions, (see [22]). For example if $T=\operatorname{Alt}(5)$, we could take $a=(123)$ and $b=(12345)$.

Lemma 4.8. Each $(\Gamma, G)$ in Construction 4.7 lies in $\mathcal{O G}(4)$ and is basic of quasiprimitive type.

Proof. First we note that $\tau$ interchanges the two elements of $S_{0}$. Consider the subgroup $N_{0}=\langle S\rangle$. The projections of $N_{0}$ to the first and second direct factors of $N$ are both equal to the group $\langle a, b\rangle=T$, since $\{a, b\}$ is a generating set for $T$. Hence either $N_{0}=N$ or $N_{0}$ is a diagonal subgroup of $N$. In the latter case there exists $\sigma \in \operatorname{Aut}(T)$ such that $N_{0}=\left\{\left(x, x^{\sigma}\right) \mid x \in T\right\}$. In particular $(a, b)=\left(a, a^{\sigma}\right)$ so $b=a^{\sigma}$, and also $(b, a)=\left(b, b^{\sigma}\right)$ so $a=b^{\sigma}$. However by assumption, no such automorphism exists. Hence $N_{0}=N$, and this implies that $\Gamma$ is connected.

We claim that $S_{0} \cap S_{0}^{-1}=\emptyset$. It is sufficient to prove that $x:=(a, b)^{-1} \notin S_{0}$. If $x=(a, b)$ then $a^{2}=b^{2}=1$ and $\langle a, b\rangle \neq T$. If $x=(b, a)$ then $b=a^{-1}$ and again $\langle a, b\rangle \neq T$. So the claim is proved and hence $\Gamma$ has valency 4 , and admits an edge orientation as described above. Then, since $N$ acts regularly on vertices by right multiplication, and since $\tau$ interchanges the two elements of $S_{0}$, it follows that $G$ is vertex-transitive and edge-transitive, and preserves the edge orientation. Hence $(\Gamma, G) \in \mathcal{O G}(4)$.

Finally, since $\tau$ acting by conjugation, interchanges the two simple direct factors of $N$, the group $N$ is a minimal normal subgroup of $G$, and is the unique minimal normal subgroup. Then since $N$ is regular on vertices, we conclude that $G$ is quasiprimitive on the vertex set of $\Gamma$. Hence $(\Gamma, G)$ is basic of quasiprimitive type.

We remark that the quasiprimitive group $G$ in Construction 4.7 is of twisted wreath type $(\mathrm{Tw})$, as defined in [38].

\section{Coset actions and coset graphs in $\mathcal{O G}(4)$}

We obtain further examples of graphs in $\mathcal{O G}(4)$ using the coset graph construction. For a group $G$, proper subgroup $H$, and element $s \in G$, the coset graph $\Gamma=\operatorname{Cos}(G, H, s)$ is the 
(undirected) graph with vertex set $V=\{H x \mid x \in G\}$, and edges $\{H x, H y\}$ if and only if $x y^{-1}$ or $y x^{-1} \in H s H$. A good account of this construction is given in [20, Section 2]. The group $G$, acting by right multiplication on $V$, induces a vertex-transitive and edgetransitive group of automorphisms of $\Gamma$. This $G$-action is faithful, so that $G \leqslant \operatorname{Aut}(\Gamma)$, if and only if $H$ is core-free, that is, $\cap_{g \in G} H^{g}=1$. The graph $\Gamma$ is $G$-oriented if and only if $s^{-1} \notin H s H$ (and otherwise $\Gamma$ is $G$-arc-transitive). In the $G$-oriented case the valency is $2\left|H: H \cap H^{s}\right|$. Also $\Gamma$ is connected if and only if $\langle H, s\rangle=G$. In summary, $(\Gamma, G) \in \mathcal{O} \mathcal{G}(4)$ if and only if

$H$ is core-free in $G, s^{-1} \notin H s H,\left|H: H \cap H^{s}\right|=2$, and $\langle H, s\rangle=G$.

Moreover, for each $(\Gamma, G) \in \mathcal{O G}(4), \Gamma=\operatorname{Cos}(G, H, s)$ for some $H, s$ satisfying (1). First we give a family of examples for nonabelian simple groups.

Construction 5.1. Let $G$ be a nonabelian simple group, and suppose that $G$ contains an element $h$ of order 2 , and an element $g$ such that $\left\{g, g^{h}\right\}$ generates $G$. Let $\Gamma=$ $\operatorname{Cos}(G,\langle h\rangle, g)$.

Lemma 5.2. For $\Gamma, G$ as in Construction 5.1, $(\Gamma, G) \in \mathcal{O G}(4)$ and is basic of quasiprimitive type.

Proof. Each coset graph for $G$ is $G$-vertex-transitive and $G$-edge-transitive. Let $H:=\langle h\rangle$. To see that $\Gamma$ is $G$-oriented observe that $g^{-1} \notin H g H=\{g, h g, g h, h g h\}$ : for if $g^{-1}=h g$ or $g h$ then $h=g^{-2}$ and so $\left\langle g, g^{h}\right\rangle$ is cyclic, and we have the same conclusion if $g^{-1}=h g h$; and if $g^{-1}=g$ then $\left\langle g, g^{h}\right\rangle$ is generated by two involutions and so is abelian or dihedral.

The fact that $\left\langle g, g^{h}\right\rangle=G$ also implies that $g^{h} \neq g$, and hence $h^{g} \neq h$, so the stabiliser $H^{g}$ of the vertex $H g$ adjacent to $H$ is not equal to $H$. Hence $H^{g} \cap H=1$ and it follows that from $(1)$ that $(\Gamma, G) \in \mathcal{O G}(4)$. Since $G$ is a nonabelian simple group, the pair is basic of quasiprimitive type.

Now we construct a family of coset graphs for the symmetric groups for which the stabilisers are unbounded. Our construction is similar in spirit to constructions of arctransitive coset graphs for $\operatorname{Sym}(n)$ in [11,34], and supplements the const ruction in [26] for the groups $\operatorname{Alt}(n)$ (see Remark 1.4(ii)).

Construction 5.3. Let $G=\operatorname{Sym}(n)$ with $n$ odd and $n \geqslant 5$, let $m:=(n-1) / 2$, let $H=\langle(i, i+m) \mid i=1, \ldots, m\rangle$, and $g=(1,2,3 \ldots, n)$. Let $\Gamma=\operatorname{Cos}(G, H, g)$.

Lemma 5.4. Let $\Gamma, G$ be as in Construction 5.3. Then $(\Gamma, G) \in \mathcal{O G}(4)$ is basic of quasiprimitive type and a vertex stabiliser $G_{x}$ has order $2^{(n-1) / 2}$.

Proof. The stabiliser of the vertex $x=H$ of $\Gamma$ is $G_{x}=H$ of order $2^{(n-1) / 2}$. To prove that $(\Gamma, G) \in \mathcal{O G}(4)$, we check that the conditions (1) hold. The only nontrivial normal subgroups of $G$ are $\operatorname{Alt}(n)$ and $G$ itself, and neither of these is contained in $H$, so $H$ is core-free in $G$. Also $H^{g}=\langle(i, i+m) \mid i=2, \ldots, m+1\rangle$, and hence $H \cap H^{g}=\langle(i, i+m)|$ $i=2, \ldots, m\rangle$ has index 2 in $H$. 
We claim that $g^{-1} \notin H g H$ : for if $g^{-1} \in H g H$ then we would have $g h g \in H$ for some $h \in H$. Since each element of $H$ fixes the point $n$ (in its natural action on $\{1,2, \ldots, n\}$ ), $n=n^{g h g}=1^{h g}=\left(1^{h}\right)^{g}$ so $1^{h}=n-1$. However the $H$-orbit containing 1 is $\{1, m+1\}$, so this is a contradiction since $n \geqslant 5$.

Finally the group $\langle H, g\rangle$ is transitive on $\{1, \ldots, n\}$, since it contains $g$, and we show that this action is primitive. Now $\langle H, g\rangle$ contains $(i, i+m)$, for each $i$, and in particular it contains $\left(1, \frac{n+1}{2}\right),\left(\frac{n+1}{2}, n\right),\left(n, \frac{n-1}{2}\right),\left(\frac{n-1}{2}, n-1\right), \ldots$ Thus any block of imprimitivity $B$ of size at least 2 , and such that $1 \in B$, must also contain $\frac{n+1}{2}, n, \frac{n-1}{2}, n-1$, and so on, it follows that $B=\{1, \ldots, n\}$. Hence $\langle H, g\rangle$ is primitive on $\{1, \ldots, n\}$, and since $\langle H, g\rangle$ contains a transposition, it must be equal to $\operatorname{Sym}(n)$. Thus the conditions (1) hold, and so $(\Gamma, G) \in \mathcal{O G}(4)$.

The only proper nontrivial normal subgroup of $G$ is $\operatorname{Alt}(n)$, and since $H$ contains odd permutations, it follows that $H \operatorname{Alt}(n)=\operatorname{Sym}(n)$, so $\operatorname{Alt}(n)$ is vertex-transitive. Thus $G$ is quasiprimitive on vertices, and so $(\Gamma, G)$ is basic of quasiprimitive type.

Finally we give a construction for quasiprimitive groups with non-simple, non-abelian minimal normal subgroups as in Theorem 1.3(c).

Construction 5.5. Let $T$ be a non-abelian simple group such that $T=\langle a, b\rangle$, where $a$ is an involution and $b^{g} \neq b a$ for any $g \in \mathbf{C}_{\text {Aut }(T)}(a)$. Consider $G:=N \rtimes\langle\iota\rangle$, where $N=T \times T$ and $\iota: N \rightarrow N$ is the automorphism defined by $(x, y)^{\iota}=(y, x)$ for every $(x, y) \in N$. Let $H=\langle(a, a), \iota\rangle, g=(b, b a)$, and $\Gamma=\operatorname{Cos}(G, H, g)$.

Explicit examples of $T, a$ and $b$ are easy to obtain: for example $T=\operatorname{Alt}(5), a=$ $(1,2)(3,4)$ and $b=(1,5,4,3,2)$.

Lemma 5.6. Let $\Gamma, G, N, H$ be as in Construction 5.5. Then $(\Gamma, G) \in \mathcal{O G}(4)$ is basic of quasiprimitive type, $N$ is the unique minimal normal subgroup of $G$, and $H=\mathbb{Z}_{2} \times \mathbb{Z}_{2}$.

Proof. We verify that the conditions in (1) hold. First it is straightforward to check that $H=\mathbb{Z}_{2} \times \mathbb{Z}_{2}$ and $H$ is core-free in $G$. Next an easy computation shows that

$$
H^{g} \cap H=\langle(a, a) \iota\rangle
$$

and hence $H^{g} \cap H$ has index 2 in $H$. To prove that $G=\langle H, g\rangle$, it is sufficient to prove that $N=\langle(a, a), g\rangle$. We argue by contradiction and we suppose that $\langle(a, a), g\rangle \leqslant M$, for some maximal subgroup $M$ of $N$. As $g=(b, b a)$ and $T=\langle a, b\rangle=\langle a, b a\rangle$, the subgroup $N$ projects surjectively onto each of the simple direct factors of $N$. Therefore $M$ is a diagonal subgroup of $N$, that is, $M=\left\{\left(x, x^{\varphi}\right) \mid x \in T\right\}$ for some $\varphi \in \operatorname{Aut}(T)$. As $(a, a) \in M$, we have $a^{\varphi}=a$ and so $\varphi \in \mathbf{C}_{\operatorname{Aut}(T)}(a)$. Moreover as $g=(b, b a) \in M$, the element $b^{\varphi}=b a$, contradicting the hypothesis in Construction 5.5. Thus $N=\langle(a, a), g\rangle$ and $G=\langle H, g\rangle$. Finally a straightforward check shows that, for none of the 16 pairs $\left(h, h^{\prime}\right)$ in $H \times H$, do we have $g^{-1}=h g h^{\prime}$. Hence $g^{-1} \notin H g H$. Thus all the conditions in (1) hold, and so $(\Gamma, G) \in \mathcal{O G}(4)$.

Since $\iota$ interchanges the two simple direct factors of $N$, it follows that $N$ is a minimal normal subgroup of $G$, and as $N$ has trivial centraliser in $G$, it is the unique minimal normal subgroup. Since $G=N H$, the group $N$ is vertex-transitive, and so $G$ is quasiprimitive 
on vertices. Thus the only $G$-normal quotient of $(\Gamma, G)$ is the one-vertex graph $K_{1}$, so $(\Gamma, G)$ is basic of quasiprimitive type.

\section{$6 \quad$ Proof of Theorem 1.3}

Suppose that $(\Gamma, G) \in \mathcal{O G}(4)$ is basic of quasiprimitive type, with vertex set $X$. Let $N$ be a minimal normal subgroup of $G$. Then $N$ is transitive on $X$ since $G$ is quasiprimitive. If $N$ is abelian then $N$ is regular (see, [38, Theorem 1]) and so by Remark 4.1, $\Gamma=$ $\operatorname{Cay}(N, S)$ for some $S$. This is not possible by Lemma 4.3. Thus $N$ is nonabelian, and so $N \cong T^{k}$ for some nonabelian simple group $T$, and integer $k \geqslant 1$. For quasiprimitive permutation groups with more than one minimal normal subgroup, the stabilisers have $T$ as a composition factor (see again [38, Theorem 1] - such groups occur as 'type III(a)' with ' $k=2$ and $P=1$ ' and 'type III(b)(ii)'). Thus, since a stabiliser $G_{x}$ is a nontrivial 2-group, $N$ is the unique minimal normal subgroup of $G$, and hence $G$ is isomorphic to a subgroup of $\operatorname{Aut}(N)=\operatorname{Aut}(T) \curlywedge \operatorname{Sym}(k)$, where $N$ is identified with its group of inner automorphisms. If $k=1$ then part (a) holds. So we may assume that $k \geqslant 2$. By [38, Theorem 1], and using the fact that $G_{x}$ is a nontrivial 2-group, there are the following two possibilities. (A subdirect subgroup of $R^{k}$, where $k \geqslant 2$, is a subgroup $H$ for which each of the $k$ projections of $H$ onto the $k$ direct factors $R$ has image equal to $R$.)

(i) $N$ is regular on $X$ (the twisted wreath case of 'type III(c)');

(ii) $N_{x}$ is a subdirect subgroup of $R^{k}$ for some nontrivial 2-subgroup $R$ of $T$ (the product case of 'type III(b)(i)').

We treat these two cases (i) and (ii) in Lemmas 6.1 and 5.6, respectively. The proof of Theorem 1.3 follows from these two lemmas.

Since $N$ is a minimal normal subgroup, $G$ permutes the $k$ simple direct factors of $N$ transitively by conjugation. Moreover, since $N$ is vertex-transitive we have $G=N G_{x}$, and hence the 2-group $G_{x}$ also permutes the $k$ simple direct factors transitively. In particular $k$ divides $\left|G_{x}\right|$.

Lemma 6.1. If $N=T^{k}$ is regular with $k \geqslant 2$, then $k=2$, the pair $(\Gamma, G)$ is as in Construction 4.7, and Theorem 1.3 (b) holds.

Proof. Since $N$ is regular, $N_{x}=1$. Then, again by $\operatorname{Remark} 4.1, \Gamma=\operatorname{Cay}(N, S)$ with $S=S_{0} \cup S_{0}^{-1}$, and $G=N \rtimes H$, where $H$ is a subgroup of $\operatorname{Aut}(N)$ which leaves $S_{0}$ invariant. By Lemma 4.2, $S_{0}=\{a, b\}$ such that $N=\langle S\rangle$ and none of $a^{2}, b^{2}, a b$ is $1_{N}$. Also $H \cong Z_{2}$ and interchanges $a$ and $b$. Since $H$ is the stabiliser of $x=1_{N}$, we saw above that $k$ divides $|H|$, and hence $k=2$. Then $a=\left(a_{1}, a_{2}\right), b=\left(b_{1}, b_{2}\right) \in N=T \times T$, and $H=\langle h\rangle$, where $h=\left(\sigma, \sigma^{\prime}\right) \tau \in \operatorname{Aut}(T)$ $2 \operatorname{Sym}(2)$, with $\sigma, \sigma^{\prime} \in \operatorname{Aut}(T)$ and $\tau=(1,2) \in \operatorname{Sym}(2)$. We require $h^{2}=1$ and as $h^{2}=\left(\left(\sigma, \sigma^{\prime}\right) \tau\right)^{2}=\left(\sigma \sigma^{\prime}, \sigma^{\prime} \sigma\right)$, it follows that $\sigma^{\prime}=\sigma^{-1}$. Next we observe that the element $(\sigma, 1) \in \operatorname{Aut}(N) \leqslant \operatorname{Sym}(N)$ induces a graph isomorphism from 
$\Gamma$ to the graph $\operatorname{Cay}\left(N, S^{(\sigma, 1)}\right)$ admitting the edge-transitive group $G^{(\sigma, 1)}=N \rtimes\left\langle h^{(\sigma, 1)}\right\rangle$. Now

$$
h^{(\sigma, 1)}=\left(\sigma^{-1}, 1\right)\left(\sigma, \sigma^{-1}\right) \tau(\sigma, 1)=\left(1, \sigma^{-1}\right) \tau(\sigma, 1)=\tau
$$

so, replacing $(\Gamma, G)$ by their images under $(\sigma, 1)$, if necessary, we may assume that $\sigma=1$, that is, $h=\tau$. Thus $G$ is as in Construction 4.7. Now $b=a^{h}=\left(a_{1}, a_{2}\right)^{\tau}=\left(a_{2}, a_{1}\right)$. Since $\Gamma$ is connected $N=\langle a, b\rangle$. In particular this implies that $\left\{a_{1}, a_{2}\right\}$ generates $T$. If there were an automorphism $\nu \in \operatorname{Aut}(T)$ that interchanged $a_{1}$ and $a_{2}$, then we would find that $\langle a, b\rangle=\left\{\left(t, t^{\nu}\right) \mid t \in T\right\}$, which is a contradiction. Hence no such automorphism exists, and $(\Gamma, G)$ is as in Construction 4.7. Thus part (b) of Theorem 1.3 holds.

Now we consider the second case above. The analysis follows closely some ideas developed in $[45,46,52,54]$. Note that examples for this case were given in Construction 5.5.

Lemma 6.2. Suppose that $N=T^{k}$ with $k \geqslant 2$, and $N_{x}$ is a subdirect subgroup of $R^{k}$ for some nontrivial 2-subgroup $R$ of $T$. Then $k=2$, and Theorem $1.3(c)$ holds.

Proof. As discussed in Subsection 3.1, $\Gamma=\mathcal{G}(\Delta)$ for some $G$-orbital $\Delta$. Let $s$ be a positive integer. By an $s$-arc of $(\Gamma, G)$ we mean a vertex-sequence $\left(x_{0}, \ldots, x_{s}\right)$ such that $\left(x_{i}, x_{i+1}\right) \in$ $\Delta$ for each $i \in\{0, \ldots, s-1\}$. Now, let $s$ be maximal such that $G$ is transitive on the set of $s$-arcs. Note that $s \geqslant 1$ since $(\Gamma, G) \in \mathcal{O G}(4)$. We claim that $G$ acts regularly on the set of $s$-arcs of $\Gamma$. Let $\left(x_{0}, \ldots, x_{s}\right)$ be an $s$-arc and consider the point-wise stabilizer $H$ in $G$ of $x_{0}, \ldots, x_{s}$. To prove the claim it suffices to prove that $H=1$. Since $G$ is not transitive on $(s+1)$-arcs, the group $H$ fixes each of the two vertices $y, y^{\prime}$ such that $\left(x_{s}, y\right),\left(x_{s}, y^{\prime}\right) \in \Delta$. (Let us call $y, y^{\prime}$ the out-neighbours of $x_{s}$.) Now $\left(x_{1}, \ldots, x_{s}, y\right)$ is an $s$-arc and so, by transitivity, there exists $g \in G$ such that $\left(x_{0}, \ldots, x_{s-1}, x_{s}\right)^{g}=\left(x_{1}, \ldots, x_{s}, y\right)$. Therefore $H^{g}=G_{x_{0}, \ldots, x_{s}}^{g}=G_{x_{1}, \ldots, x_{s}, y}$. However, since $H$ fixes $y$ it follows that $G_{x_{1}, \ldots, x_{s}, y} \leqslant H$, and hence $H^{g}=H$. The fact that $H$ fixes the two out-neighbours of $x_{s}$ implies that $H^{g}$ fixes the two out-neighbours of $x_{s}^{g}=y$, that is to say, $H=H^{g}$ fixes also the two out-neighbours of $y$. At this point it is clear that a quick inductive argument (using the fact that $\Gamma$ is connected) yields that $H=1$. Thus the claim is proved.

Since $G$ is regular on $s$-arcs, its subgroup $G_{x_{0}}$ is regular on the set of $s$-arcs starting at $x_{0}$. From this it quickly follows that, for each $i \in\{0, \ldots, s\}$, the subgroup $G_{x_{0}, \ldots, x_{s-i}}$ has order $2^{i}$. In particular, $\left|G_{x_{0}, \ldots, x_{s-1}}\right|=2$, say $G_{x_{0}, \ldots, x_{s-1}}=\left\langle h_{1}\right\rangle$. Let $g \in G$ be as in the previous paragraph and set $x_{s+1}=y$. For $i \in\{2, \ldots, s\}$, define $h_{i}=h_{i-1}^{g^{-1}}$. It is clear that, for each $i$, we have

$$
G_{x_{0}, \ldots, x_{s-i}}=\left\langle h_{1}, \ldots, h_{i}\right\rangle .
$$

As elements of $\operatorname{Aut}(N)=\operatorname{Aut}(T) \imath \operatorname{Sym}(k), h_{1}$ and $g$ may be written as $h_{1}=f \sigma$ and $g=f^{\prime} \tau$ with $f, f^{\prime} \in \operatorname{Aut}(T)^{k}$ and $\sigma, \tau \in \operatorname{Sym}(k)$. Note that, since $h_{1}^{2}=1$, we also have $\sigma^{2}=1$. Let $\pi$ denote the projection map $\operatorname{Aut}(N) \rightarrow \operatorname{Sym}(k)$, so that $\left(h_{1}\right) \pi=\sigma$, and $(g) \pi=\tau$. Now $K:=(G) \pi=\left(N G_{x_{0}}\right) \pi=\left(G_{x_{0}}\right) \pi$ is a 2-group since $G_{x_{0}}$ is a 2-group. Moreover,

$$
K=\left(G_{x_{0}}\right) \pi=\left\langle h_{1}, h_{2}, \ldots, h_{s}\right\rangle \pi=\left\langle\sigma, \sigma^{\tau^{-1}}, \sigma^{\tau^{-2}}, \ldots, \sigma^{\tau^{-(s-1)}}\right\rangle
$$


We claim that $K=\langle\sigma\rangle$, from which it follows that $k=2$ since $\sigma^{2}=1$. Suppose to the contrary that $\langle\sigma\rangle$ is a proper subgroup of $K$, and let $M$ be a maximal subgroup of $K$ containing $\sigma$. As $K$ is a 2-group, $M$ is normal in $K$ and hence $\sigma^{\tau^{-i}} \in M$, for all $i \in \mathbb{Z}$. It then follows from (2) that $K \leqslant M$, whence $K=M$, which is a contradiction. Thus $K=\langle\sigma\rangle \cong \mathbb{Z}_{2}$ and $k=2$.

\section{Acknowledgements}

The authors are grateful to Gabriel Verret for reading the draft manuscript and for his advice on exposition and related work. We thank Roman Nedela for his interest in our research and advice on references. We thank an anonymous referee for helpful feedback. This project was funded by the Deanship of Scientific Research (DSR), King Abdulaziz University, Jeddah, under grant no. HiCi/H1433/363-1. The authors, therefore, acknowledge with thanks DSR technical and financial support.

\section{References}

[1] J. A. Al-bar, A. N. Al-kenani, N. M. Muthana, and C. E. Praeger, Finite edgetransitive oriented graphs of valency four with cyclic normal quotients. Preprint, 2015.

[2] J. A. Al-bar, A. N. Al-kenani, N. M. Muthana, and C. E. Praeger, A normal quotient analysis for some families of oriented four-valent graphs. Submitted 2015.

[3] E. Bannai, R. L. Griess, C. E. Praeger, and L. Scott, The Mathematics of Donald Gordon Higman, Mich. Math. J. 58 (2009), 3-30.

[4] J. van Bon, Finite primitive distance-transitive graphs, Eur. J. Combin. 28 (2007), 517-32.

[5] I. Z. Bouwer, Vertex and edge-transitive but not 1-transitive graphs, Canad. Math. Bull. 13 (1970), 231-237.

[6] P. J. Cameron, Permutation groups. London Math. Soc. Student Texts, Cambridge University Press, Cambridge, 1999.

[7] P. J. Cameron, C. E. Praeger, N. C. Wormald,Infinite highly arc transitive digraphs and universal covering digraphs, Combinatorica 13 (1993) 377-396.

[8] M. D. E. Conder and D. Marušič, A tetravalent half-arc-transitive graph with nonabelian vertex stabilizer, J. Combin. Theory Ser. B 88 (2003), 67-76.

[9] M. D. E. Conder and J. Ma, Arc-transitive abelian regular covers of cubic graphs, J. Algebra 387 (2013), 215-242.

[10] M. D. E. Conder, P. Potočnik, and P. Šparl, Some recent discoveries about half-arctransitive graphs, Ars Math. Contemp. 8 (2015), 149-162.

[11] M. D. E. Conder and C. G. Walker, Vertex-transitive non-Cayley graphs with arbitrarily large vertex-stabilizer, J. Algebraic Combin. 8 (1998), 29-38. 
[12] W. Dyck, Uber Aufstellung und Untersuchung von Gruppe und Irrationalitat regularen Riemannscher Flachen, Math. Ann. 17 (1880), 473-508.

[13] A. Gardiner and C.E.Praeger, On 4-valent symmetric graphs, Eur. J. Combin. 15 (1994), 375-381.

[14] G. Glauberman, Normalizers of p-subgroups in fnite groups, Pacific J. Math. 29 (1969), 137-144.

[15] C. Godsil and G. F. Royle, Algebraic Graph Theory, Springer-Verlag, New York, 2001.

[16] G. Jones and D. Singerman, Theory of maps on orientable surfaces, Proc. London Math. Soc. 37 (1978), 273-307.

[17] D. G. Higman, Intersection matrices for finite permutation groups, J. Alg. 6 (1967), $22-42$.

[18] A. Hujdurović, K. Kutnar and D. Marušič, Half-arc-transitive group actions with a small number of alternets, J. Combin. Theory, Ser. A 124 (2014), 114-129.

[19] K. Kutnar, D. Marušič, P. Šparl, R.-J. Wang, M.-Y. Xu, Classification of half-arctransitive graphs of order 4p, Eur. J. Combin. 34 (2013), 1158-1176.

[20] C. H. Li, Z. P. Lu and D. Marušič, On primitive permutation groups with small suborbits and their orbital graphs, J. Algebra 279 (2004), 749-770.

[21] C. H. Li, C. E. Praeger, A. Venkatesh, and S. Zhou, Finite locally-quasiprimitive graphs, Disc. Math. 246 (2002), 197-218.

[22] M. W. Liebeck and A. Shalev, Random $(r, s)$-generation of finite classical groups. Bull. London Math. Soc. 34 (2002), 185-188.

[23] A. Malnič, D. Marušič and P. Potočnik, Elementary abelian covers of graphs, J. Algebraic Combin. 20 (2004), 71-97.

[24] D. Marušič, Recent developments in half-transitive graphs, Discrete Math 182 (1998), 219-231.

[25] D. Marušič, Half-transitive group actions on finite graphs of valency 4, J. Combin. Theory (B) 73 (1998), 41-76.

[26] D. Marušič, Quartic half-arc-transitive graphs with large vertex stabilizers, Discrete Math 299 (2005), 180-193.

[27] D. Marušič and R. Nedela, Maps and half-transitive graphs of valency 4, Eur. J. Combin. 19 (1998), 345-354.

[28] D. Marušič and R. Nedela, On the point stabilizers of transitive groups with nonself-paired suborbits of length 2, J. Group Theory 4 (2001), 19-43.

[29] D. Marušič and C. E Praeger. Tetravalent graphs admitting half-transitive group actions: alternating cycles, J. Combin. Theory (B) 75 (1999), 188-205.

[30] D. Marušič and P. Sparl, On quartic half-arc-transitive metacirculants. J. Alg. Combin. 28 (2008), 365-395. 
[31] D. Marušič and A. O. Waller, Half-transitive graphs of valency 4 with prescribed attachment numbers, J. Graph Theory 34 (2000), 89-99.

[32] D. Marušič and M. Y. Xu, A 1/2-transitive graph of valency 4 with a nonsolvable group of automorphisms, J. Graph Theory 25 (1997), 133-138.

[33] P. Potočnik and R. Požar. Smallest tetravalent half-arc-transitive graphs with vertexstabiliser isomorphic to the dihedral group of order 8. J. Combin. Theory Series A, to appear.

[34] P. Potočnik, P. Spiga and G. Verret, Tetravalent arc-transitive graphs with unbounded vertex-stabilizers. Bull. Aust. Math. Soc. 84 (2011), 79-89.

[35] P. Potočnik, P. Spiga and G. Verret, A census of 4-valent half-arc-transitive graphs and arc-transitive digraphs of valence two. Ars Math. Contemporanea 8 (2015), 133148.

[36] P. Potočnik and G. Verret, On the vertex-stabiliser in arc-transitive digraphs, J. Combin. Theory Series B 100 (2010), 497-509.

[37] C. E. Praeger, Highly arc transitive digraphs, Europ. J. Combin. 10 (1989), 281-292.

[38] C. E. Praeger, An O'Nan-Scott Theorem for finite quasiprimitive permutation groups, and an application to 2-arc transitive graphs, J. London Math. Soc. (2) 47 (1993), 227-239.

[39] C. E. Praeger (with the assistance of C. H. Li and A. C. Niemeyer), Finite transitive permutation groups and finite vertex-transitive graphs, in Graph Symmetry: Algebraic Methods and Applications, Eds: Gena Hahn and Gert Sabidussi, NATO ASI Ser.C 497, KLuwer, 1997. pp. 277-318.

[40] C. E. Praeger, Finite transitive permutation groups and bipartite vertex-transitive graphs, Illinois J. Math. 47 (2003), 461-475.

[41] C. E. Praeger, L. Pyber, P. Spiga, and E. Szabó, Graphs with automorphism groups admitting composition factors of bounded rank. Proc. Amer. Math. Soc. 140 (2012), 2307-2318.

[42] C. E. Praeger, J. Saxl and K. Yokohama, Distance transitive graphs and finite simple groups, Proc. London Math. Soc. (3) 55 (1987), 1-21.

[43] G. Sabidussi, Vertex-transitive graphs, Monatsh. Math. 68 (1964), 426-438.

[44] Ákos Seress, Toward the classification of $s$-arc transitive graphs. Groups St. Andrews 2005. Vol. 2, London Math. Soc. Lecture Note Ser., 340, Cambridge Univ. Press, Cambridge, 2007, pp. 401-414.

[45] C. C. Sims, Graphs and finite permutation groups, Math. Zeit. 95 (1967), 76-86.

[46] C. C. Sims, Graphs and finite permutation groups. II, Math. Zeit. 103 (1968), 276281.

[47] P. Spiga and G. Verret, On the order of vertex-stabilisers in vertex-transitive graphs with local group $C_{p} \times C_{p}$ or $C_{p}$ $C_{2}$, J. Algebra 448 (2016), 174-209. 
[48] P. Šparl, A classification of tightly attached half-arc-transitive graphs of valency 4, J Combin Theory Ser B 98 (2008), 1076-1108.

[49] G. Szekeres, Polyhedral decompositions of cubic graphs Bull. Austral. Math. Soc. 8 (1973), 367-387.

[50] J. Tits, Algebraic and Abstract Simple Groups, Annals of Math. Second Series, 80 (1964), 313-329.

[51] J. Tits, Buildings of spherical type and finite BN-pairs. Lecture Notes in Mathematics. 386. Berlin-Heidelberg-New York, Springer-Verlag, 1974.

[52] W. T. Tutte, A family of cubical graphs, Proc. Camb. Phil. Soc. 43 (1947), 459-474.

[53] W. T. Tutte, On the imbedding of linear graphs in surfaces. Proc. London Math. Soc. (2) $51,(1949) .474-483$.

[54] W. T. Tutte, On the symmetry of cubic graphs, Canad. J. Math. 11 (1959), 621-624.

[55] W. T. Tutte, A census of planar maps, Can. J. Math. 15 (1963), 249-271.

[56] W. T. Tutte, Connectivity in graphs, University of Toronto Press, Toronto, 1966.

[57] S. Wilson, Semi-transitive graphs, J. Graph Theory 45 (2004), 1-27. 